\title{
Experimental Link Quality Characterization of Wireless Sensor Networks for Underground Monitoring
}

\author{
Bruno Silva, Student Member, IEEE, Roy M. Fisher, Student Member, IEEE, \\ Anuj Kumar, and Gerhard P. Hancke, Senior Member, IEEE
}

\begin{abstract}
Wireless underground sensor networks (WUSNs) are a category of wireless sensor networks (WSNs) with buried nodes, which communicate wirelessly through soil with sensor nodes located aboveground. As the communication medium (i.e., soil) between traditional over-the-air WSNs and WUSNs differs, communication characteristics have to be fully characterized for WUSNs, specifically to enable development of efficient communication protocols. Characterization of link quality is a fundamental building block for various communication protocols. The aim of this paper is to experimentally investigate the link quality characteristics of the three communication channels available in WUSNs for underground pipeline monitoring to gain further insight into protocol development for WUSNs. To this end, received signal strength (RSS), link quality indicator (LQI), and packet reception ratio (PRR) are characterized for the three communication channels in WUSNs. The RSS and PRR results show that the underground-to-underground channel is highly symmetric and temporally stable, but its range is severely limited, and that the aboveground-to-underground/undergroundto-underground channels are asymmetric and exhibit similar temporal properties to over-the-air communication channels. Interestingly, the results show that RSS is a better indicator of PRR than LQI for all three channels under consideration.
\end{abstract}

Index Terms-Communication, industrial, link quality, underground, wireless sensor networks (WSNs).

\section{Introduction}

Recent advances in microelectro-mechanical systems (MEMS) have led to the rise of ubiquitous computing and wireless sensor networks (WSNs). WSNs enable a wide array of applications including localization [1], environmental monitoring [2]-[4], habitat monitoring [5], and monitoring in various harsh industrial environments [6]-[8]. The majority of these applications only require the use of the wireless over-the-air (OTA) channel, which is common to most currently deployed

B. Silva and A. Kumar are with the Department of Electrical, Electronic, and Computer Engineering, University of Pretoria, Pretoria 0002, South Africa (e-mail: anuj.kumar@up.ac.za).

R. M. Fisher is with the Department of Electrical, Electronic, and Computer Engineering, University of Pretoria, Pretoria 0002, South Africa, and also with the Department of Computer Science, City University of Hong Kong, Hong Kong (e-mail: rmfisher@ cityu.edu.hk).

G. P. Hancke is with the Department of Computer Science, City University of Hong Kong, Hong Kong (e-mail: gp.hancke@ city.edu.hk).
WSNs. There are a number of monitoring applications which can benefit from sensor nodes deployed underground, such as underground pipeline monitoring and precision agriculture, where buried nodes communicate wirelessly through soil with sinks located aboveground. From an industrial perspective, since WSNs enable monitoring in various environments, it is of interest to determine the link characteristics for environments such as factories [9]-[11], and also to investigate efficient ways to integrate WSNs and Internet of Things into existing industrial network infrastructure [12]-[15]. Reliable data delivery, in particular, is a major concern in industrial settings [16]. For example, characterizing the link quality for pipeline monitoring wireless underground sensor networks (PM-WUSNs) is a natural first step toward development of efficient PM-WUSNs for industrial applications.

Pipelines are used worldwide to transport gases and liquids over long distances (often over thousands of kilometers) and faults can lead to catastrophic and costly consequences; therefore, is it imperative to reliably monitor pipelines to alert entities of any anomaly in a timely manner. Several sensor network solutions exist for aboveground pipelines in contrast to underground pipelines. The distinct difference between WSNs for aboveground monitoring and WUSNs is the communication medium. Buried sensor nodes can only communicate wirelessly through soil and therefore face a number of challenges. First, soil is a mixture of organic matter, minerals, and water, and the path loss in soil is much higher than in free space. The amount of path loss is dependent on the soil properties and conditions, which can be affected by rain or by artificial irrigation. Second, since sensor nodes are buried and are not easily accessible for battery replacements, energy efficiency has to be maximized. A bulk of wasted energy in WSNs can be attributed to packet retransmissions so link characterization is essential for WUSNs, as understanding link characteristics is vital to the development of efficient communication protocols. Propagation models available for traditional WSNs are not applicable to WUSNs. There has been some work on WUSN channel characterization [17]-[21], but to the best of our knowledge, there has been no work which has experimentally analyzed link quality characteristics for WUSNs specifically.

Link quality is a fundamental building block for a range of communication protocols in WSNs [22]. As the high path loss coupled with the relatively weak signals transmitted by low power radios, and the effects of nonisotropic 


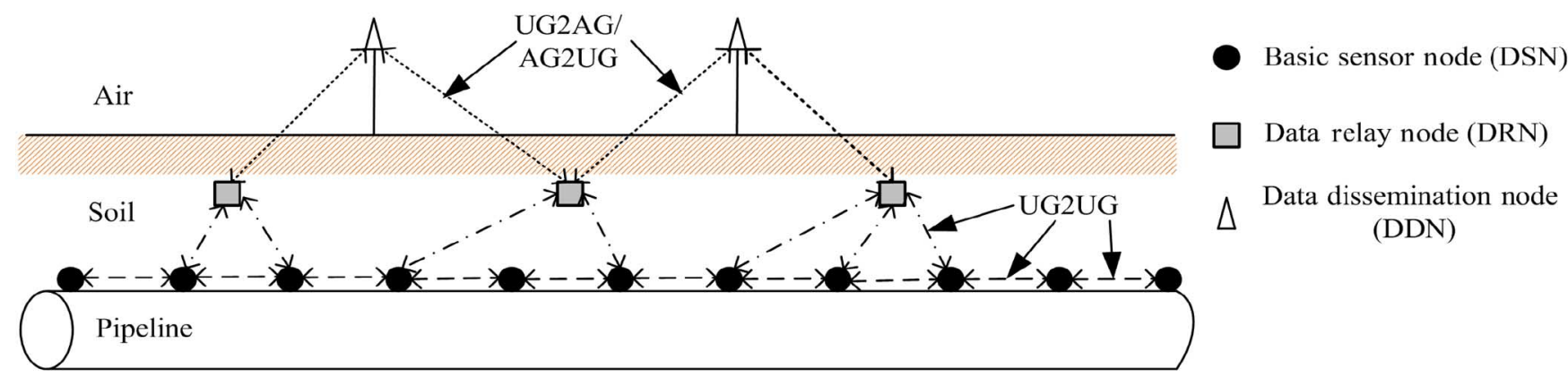

Fig. 1. WUSN for underground pipeline monitoring.

antennas radiation patterns [23] contribute toward making wireless communication in the underground medium difficult, link quality characterization is vital for efficient protocol development for WUSNs. Communication in WUSNs takes place over three different wireless channels: the underground-tounderground (UG2UG) channel is used for communication between two buried nodes; the underground-to-aboveground (UG2AG) channel is used for communication between a buried node and an aboveground node (typically a sink node); and the aboveground-to-underground (AG2UG) channel is used for communication between an aboveground node and a buried node. These channels are illustrated in Fig. 1.

In this paper, we analyze the link quality characteristics for the three channels in WUSNs, by considering the temporal and spatial characteristics of received signal strength (RSS), link quality indicator (LQI), and packet reception ratio (PRR), as well as link symmetry in both dry and wet soil conditions. Several studies have investigated link quality for aboveground WSNs [24] and it is well known that the RSS is easily accessible in most radio frequency (RF) transceivers and it is therefore the simplest metric for link quality evaluation [24]. However, it has been shown that RSS is not the most reliable metric, especially if it is sampled very close to the receiver sensitivity [25].

LQI is highly variant and it is slow to adapt to rapid link changes since it requires a large estimation window (due to its high variance). Nevertheless, its high variance has been successfully exploited for fast link quality assessment [26] and mean LQI has also been shown to have a high correlation with PRR [9], [24]. In contrast to RSS, PRR is an unambiguous metric since it can reflect the actual link quality, as high interference can result in a high RSS, leading to an erroneous high-quality assessment for a link which might actually have a low PRR [27]. A holistic characterization of link quality using the three metrics is preferable to using only a single metric; therefore, this study considers all three metrics. Of particular importance is also the relationship (i.e., correlation) between PRR, LQI, and RSS. It is important to understand this relationship because both LQI and RSS are hardware-based estimators, and therefore are easily determined. Hence, if these relationships are well understood, it is then possible to use RSS or LQI as indicators of PRR, avoiding the "costly" operation of directly estimating PRR, and therefore improving energy consumption in WUSNs. In a number of studies for conventional WSNs, it has been shown that mean LQI is a better indicator of PRR than mean RSS (the reader is referred to [24] for more information); therefore, it is worth investigating whether this is also the case in WUSNs.

To the best of our knowledge, none of the experimental studies in literature have considered the three communication channels in WUSNs in a unified manner. Most studies either investigated link characteristics of the UG2UG channel, or AG2UG/UG2AG channels, separately. This poses a challenge because nodes in practical deployments of WUSNs will ideally only be equipped with one antenna (due to cost and power constraints), so it is crucial to determine the link quality characteristics of all three channels with the same antenna configuration, to get a better perspective into the link behavior in realistic scenarios.

Without analyzing link quality characteristics, it is not possible to develop efficient communication protocols; therefore, further insight into link quality characteristics in WUSNs is required. In this investigation, the link quality characteristics of all three channels are determined with a unique experimental setup (i.e., antenna and transceiver configuration). The contributions of this paper are as follows.

1) This is the first study, to the best of our knowledge, which characterizes the link quality in all three communication channels in a unified manner using the same experimental setup.

2) This allows for an analysis of the relationship between PRR, LQI, and RSS for WUSNs using experimental data.

While previous studies analyzed some of the link quality characteristics of WUSNs, our work delves deeper into the relationship between multiple metrics, searching for insight into the intricacies of link quality characteristics. This work enables researchers to develop more energy-efficient communication protocols specifically suited for WUSNs, which exploit the relationship between characteristics of link quality metrics, which was not possible with previous studies. We consider a scenario where sensor nodes are deployed along the outer surface of a pipeline (i.e., out-of-pipe monitoring) and communication between all sensor nodes occurs through soil. Our analysis is supported by experiments conducted in an experimental farm. The results of this study will help researchers understand the intricacies of link quality in the three communication channels under consideration, such that more efficient communication protocols can be implemented for WUSNs. Although experiments are not conducted in an actual pipeline environment, the scenarios presented in this paper are representative of an 


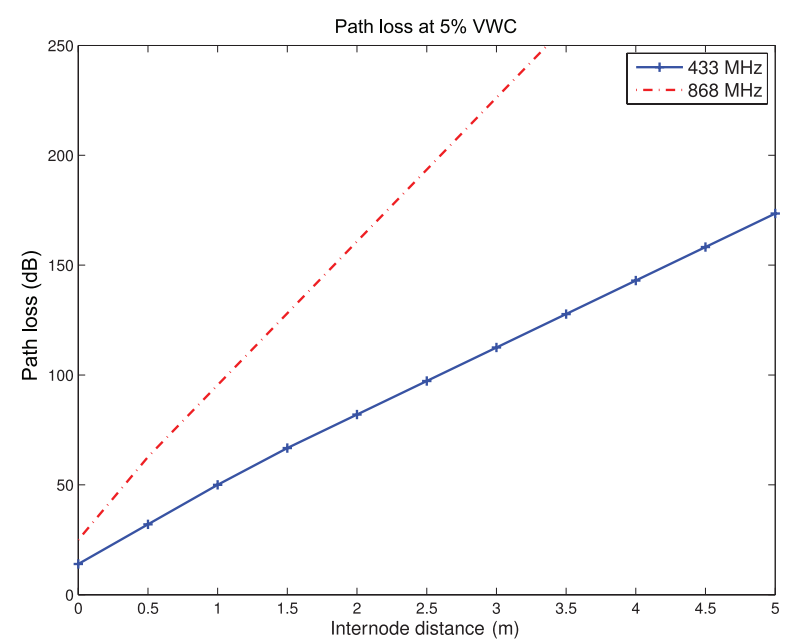

Fig. 2. Path loss for 433 and $868 \mathrm{MHz}$ at $5 \%$ VWC.

employed. Furthermore, the broad scope of our work is on link quality characterization of WUSNs, of which PM-WUSNs are a good example of. A typical PM-WUSN is illustrated in Fig. 1, where a hierarchical topology which consists of basic sensor nodes (BSNs), data relay nodes (DRNs), and data dissemination nodes (DSNs), is illustrated, as proposed in [28].

This paper is organized as follows. Section II introduces some theoretical concepts necessary for the calculation of parameters used in experiments. Section III discusses the experimental setup and protocol. Section IV discusses the results. Section V discusses related work and how this work builds on literature. In Section VI, this paper is finally concluded.

\section{Background}

This section introduces some background theory and equations to our work. Propagation in soil differs from propagation in air, and wavelengths are affected by soil properties and conditions. To effectively conduct our work, we rely on previous work in literature to estimate soil and antenna parameters.

\section{A. Calculation of Soil-Related Parameters}

Electromagnetic (EM) propagation characteristics in dielectric mediums such as soil are dependent on the medium's properties. In air, parameters such as temperature and humidity have no significant effect on communication quality (except for extremely high-frequency wireless communication). In soil, however, propagation characteristics are dependent on soil conditions and properties. Fig. 2 shows the path loss over a UG2UG link for two center frequencies (433 and $868 \mathrm{MHz}$ ), at a volumetric water content (VWC) of $5 \%$.

Soil is a nonhomogeneous dielectric substance; therefore, its propagation properties are dependent on its conductivity, permeability, and permittivity [29], [30]. For instance, an increase in soil's VWC results in an increase in its relative permittivity and conductivity, which consequently results in higher path loss. To characterize EM propagation in soil, a model which relates the soil properties and conditions to EM propagation characteristics is required. One such model is Peplinski's model [31], which is used to estimate the soil's dielectric constant (for frequencies in the $0.3-1 \mathrm{GHz}$ band) based on the sand percentage, clay percentage, volumetric water content, soil particle specific density, and soil bulk density. The dielectric constant of the soil's solids $\epsilon_{s}$ is given by

$$
\epsilon_{s}=\left(1.01+0.44 \rho_{s}\right)^{2}-0.062 \text {. }
$$

The real and imaginary parts of the complex soil's dielectric constant can then be computed as

$$
\begin{gathered}
\epsilon_{I}=115\left[1+\frac{\rho_{b}}{\rho_{s}}\left(\epsilon_{s}^{\alpha}\right)+m_{v}^{\beta_{I}} \epsilon_{f_{w}}^{I \alpha}-m_{v}\right]^{\frac{1}{\alpha}}-0.68 \\
\epsilon_{I I}=\left[m_{v}^{\beta_{I I}} \epsilon_{f_{w}^{I I \alpha}}^{I I \alpha}\right]^{\frac{1}{\alpha}}
\end{gathered}
$$

where $\rho_{b}$ is the specific density of soil particles, $\rho_{s}$ is the soil bulk density, $m_{v}$ is the soil's VWC, $\alpha$ is 0.65 (empirically determined), $\epsilon_{f_{w}}^{I}$ and $\epsilon_{f_{w}}^{I I}$ are the real and imaginary parts of the relative dielectric constant of free water. $\beta_{I}$ and $\beta_{I I}$ are empirically determined parameters from the sand and clay percentages of soil [31], given by

$$
\begin{gathered}
\beta_{I}=1.2748-0.519 S-0.152 C \\
\beta_{I I}=1.3379-0.603 S-0.166 C
\end{gathered}
$$

where $S$ is the sand percentage and $C$ is the clay percentage. Since this paper considers communication at $433 \mathrm{MHz}$, Peplinski's model is used to calculate the required parameters using (2)-(5).

\section{B. Calculation of Antenna Frequencies}

Soil's refractive index (which is typically larger than that of air) affects the wavelength of EM waves propagating in soil. Since the soil's relative permittivity changes with VWC, an antenna which is able to accommodate a wide range of frequencies is required so that it can adapt to different soil conditions. The relative permittivity (i.e., dielectric constant) is related to the real and imaginary parts of the complex permittivity by

$$
\epsilon_{r}=\frac{\sqrt{\epsilon_{I}^{2}+\epsilon_{I I}^{2}}+\epsilon_{I}}{2 \mu_{r}}
$$

where the relative permeability $\mu_{r}$ is approximately 1 for most soils (i.e., most soils are typically nonmagnetic). Therefore, using $\epsilon_{I}$ and $\epsilon_{I I}$, which are calculated in (2) and (3), respectively, $\epsilon_{r}$ can be computed in (6). Then, the wavelength of a wave propagating in soil is calculated as

$$
\lambda=\frac{c_{0}}{f \sqrt{\epsilon_{r}}}
$$

where $\lambda$ is the wavelength, $c_{0}$ is the speed of light in vacuum, $f$ is the RF, and $\epsilon_{r}$ is the soil's relative permittivity. For OTA communication, $\epsilon_{r} \approx 1$, hence antennas designed for aboveground wireless communication are not adequate for underground usage, since the relationship between frequency and wavelength in soil is not the same as in air. Therefore, in this paper, (2)-(7) are used to compute antenna frequency bounds for our experimental work (i.e., minimum and maximum frequencies for 


\section{Experimental Setup}

Experiments were conducted at the University of Pretoria's experimental farm. The goal of these experiments was to determine the link quality characteristics of links in UG2UG, AG2UG/UG2AG channels, in both wet and dry soil conditions. To this end, there are a few preliminary tasks that are necessary, such as soil characterization, transceiver configuration, and antenna selection, which are all described in this section, along with the experimental protocol. In particular, the comparison between dry and wet conditions allows us to determine if the difference in VWC has an impact on link quality characteristics, and if so, what the actual impact is. The three metrics (RSS, LQI, and PRR) are collected using the described experimental setup, and are then analyzed offline for their temporal and spatial characteristics. The correlations between RSS and PRR, as well as LQI and PRR, are also investigated to determine whether RSS and LQI are good indicators of PRR in WUSNs. It is of interest to determine what the characteristics are, whether RSS and LQI are good indicators of PRR, and whether their behavior differs in comparison to conventional WSNs. The experimental setup described here will aid in achieving this goal.

A difference in season would affect the soil's moisture content over the course of WUSN deployment. It is, therefore, important to evaluate the link quality for both dry and wet scenarios that simulates the dynamic environmental conditions which can occur over a long-term period. Instead of a longterm study (i.e., over several months) that relies on the natural weather to induce dry and wet soil conditions we chose to work in dry conditions and to induce wet conditions. The sensor nodes were deployed in an experimental agricultural lot at our institution's teaching farm, which is used to study the effects of rainfall and irrigation on soil conditions. The plot is contained by barriers to prevent water run-off and allows for the soil to be flooded in a controlled manner, i.e., water added to the plot seeps into the intended soil area, so by controlling the amount of water, the soil moisture content can be controlled and if needed consistently recreated. Our experiments were conducted at the end of the dry season, which enabled us to work in dry conditions and allowed the experimental plot to quickly dry out if we wanted to repeat the experiments. The wet conditions were created by controlled flooding of the experimental site with water for $45 \mathrm{~min}$ and conducting the experiments approximately $24 \mathrm{~h}$ later after, allowing the water to settle throughout the soil.

\section{A. Node Deployment and Topology}

A number of sensor nodes were deployed on a small plot and soil samples were collected at the site for characterization. The experimental plot is shown in Fig. 3. Multiple holes were dug at the experimental site, with interhole distances of $0.4,0.8,1.25$, 2 , and $2.5 \mathrm{~m}$ so that various UG2UG links could be tested. The hole topology is illustrated in Fig. 4. These holes were dug to a maximum depth of $40 \mathrm{~cm}$. This topology is preferred over a conventional grid setup (as used in [26]) because holes in any

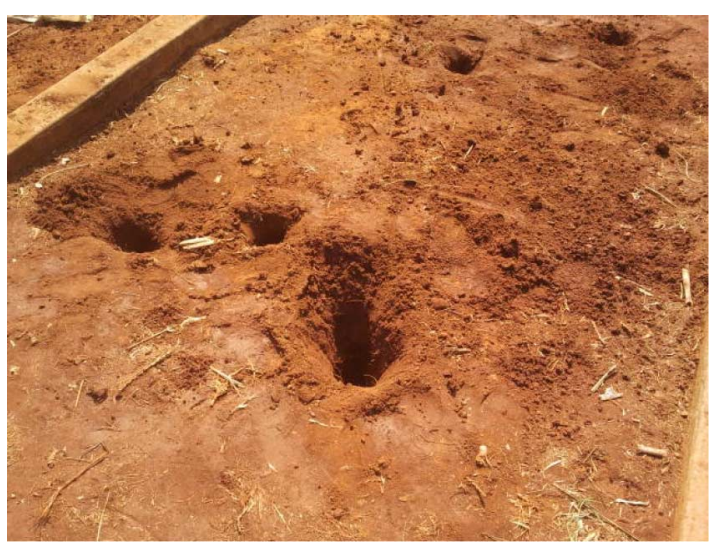

Fig. 3. Experimental site.

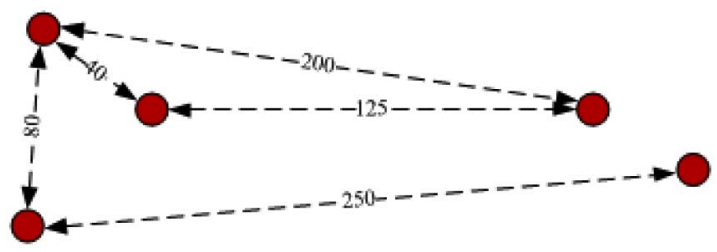

Fig. 4. Hole topology.

TABLE I

TransceIVER CONFIGURATION

\begin{tabular}{l||c} 
Parameter & Specification \\
\hline Modulation & 2-FSK \\
\hline Data rate & $1.2 \mathrm{kbaud}$ \\
\hline RX filter bandwith & $58 \mathrm{kHz}$ \\
\hline Frequency deviation & $5.4 \mathrm{kHz}$ \\
\hline Centre frequency & $433 \mathrm{MHz}$ \\
\hline TX power & $10 \mathrm{dBm}$ \\
\hline Payload length & $30 \mathrm{bytes}$
\end{tabular}

UG2UG path can influence the communication performance since the signal might cross multiple soil to air and air to soil interfaces.

\section{B. Transceiver Configuration}

Motes equipped with TI CC430 [32] transceivers operating at $433 \mathrm{MHz}$ were used for all experiments. As shown in Fig. 2, waves at $433 \mathrm{MHz}$ undergo less attenuation than waves at $868 \mathrm{MHz}$ or $2.4 \mathrm{GHz}$. The transceivers were configured for maximum receiver sensitivity, such that even severely attenuated signals could still be detected by the receiver. Table I contains the transceiver configuration.

\section{Soil Characterization}

Four soil samples were collected at the experimental site and analyzed for sand, silt, clay, and VWC percentages. The soil samples correspond to four scenarios: dry topsoil (i.e., $20 \mathrm{~cm}$ depth), wet topsoil, dry subsoil (i.e., $40 \mathrm{~cm}$ depth), and wet subsoil. The VWC was determined with the oven-drying method, which consisted of drying the samples for $24 \mathrm{~h}$ at a temperature 
TABLE II

RESUlts of SOIL SAMPles ANALySis

\begin{tabular}{|c|c|c|c|c|c|}
\hline Depth (cm) & Sand (\%) & Silt (\%) & Clay (\%) & Dry VWC (\%) & Wet VWC (\%) \\
\hline 20 & 72.9 & 19 & 8.1 & 1.31 & 20.63 \\
\hline 40 & 58 & 33 & 8.9 & 10.85 & 22.25 \\
\hline
\end{tabular}

of $100^{\circ} \mathrm{C}$. Taking into account the sample's mass before and after the drying process, the VWC is computed as

$$
\operatorname{VWC}(\%)=\left(\frac{w m-d m}{d m}\right) 100
$$

where $d m$ denotes soil's mass (in grams) before drying and $w m$ denotes soil's mass after drying. Table II shows the soil characteristics for the soil samples.

From the results in Table II, it is seen that the soil at the experimental site is predominantly sandy.

\section{Antenna Selection}

Based on the soil sample results in Table II, the upper and lower wavelengths of the buried antennas are calculated using (2)-(7), and then used to determine antenna frequency bounds (i.e., the highest and lowest supported frequencies of the antenna). Based on the data in Table II, the lower and upper wavelengths for the worst (i.e., wet soil with the largest clay percentage) and best (i.e., dry soil with the least amount of clay) cases were found to correspond to relative permittivity values of 19.3 and 5.24, which in turn correspond to wavelengths of 15.77 and $30.27 \mathrm{~cm}$, respectively. Antennas for OTA communication which correspond to these wavelengths have frequencies of $991 \mathrm{MHz}$ and $1.9 \mathrm{GHz}$, respectively. Since experiments were only conducted in soil with properties as listed in Table II and no long-term deployment was required, a GSM dual-band antenna that covers these frequencies was selected.

To determine whether these antennas were actually an improvement over $433-\mathrm{MHz}$ antennas, a simple test on a 40-cm UG2UG link was performed with buried nodes equipped with 3-dBi dipole 433-MHz antennas, and then the same test was repeated with GSM antennas. The average RSS at the receiver with the 433-MHz antennas was found to be less than $-85 \mathrm{dBm}$, whereas with the GSM antennas, it was found to be close to $-62 \mathrm{dBm}$. This result demonstrates the effectiveness of the GSM antenna over traditional 433-MHz antennas. It is noted that in previous studies, such as [17] and [33], this phenomenon was not considered, and $433-\mathrm{MHz}$ antennas were used for UG2UG experiments.

\section{E. Experimental Protocol}

Three sensor nodes equipped with Texas Instruments CC430 radios are used: one node placed above ground equipped with a $3-\mathrm{dBi} 433-\mathrm{MHz}$ antenna and two nodes buried in soil equipped with dual-band GSM antennas. The aboveground node is connected to a laptop (for data logging and coordination of experiments) which serves as the interface to the system allowing a user to trigger the start of experiments.

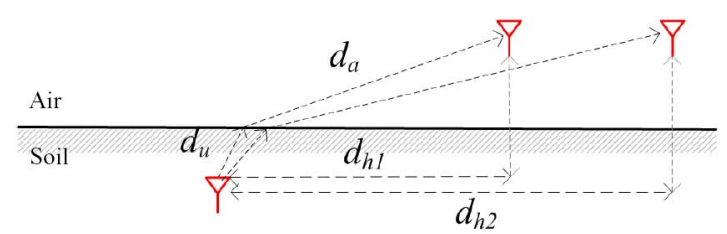

Fig. 5. Experimental scenario for UG2AG/AG2UG.

Burial depths of 20 and $40 \mathrm{~cm}$ (where the burial depth is measured from the tip of the antenna to the soil surface) are used for experiments. Various links (as illustrated in Fig. 4) were tested in both wet and dry scenarios. The three nodes allowed testing of the UG2UG, AG2UG, and UG2AG channels as follows. Assuming nodes 1 and 2 are buried (and node 3 is the aboveground sink), the UG2UG channel is tested by setting node 1 as a source node which continuously transmits packets until the packet count reaches 500. The RSS and LQI of the packets received at node 2 (which serves as a relay node) are extracted and inserted into the payload of new packets, which are then transmitted from nodes 2 to 3 . Upon receiving a packet from node 2, the LQI and RSS in the payload (which correspond to the statistics for the UG2UG link between nodes 1 and 2) are logged and analyzed at node 3 . The PRR is then determined as the number of packets received with a cyclic redundancy check (CRC) of 1, divided by 500. Reversing the roles of nodes 1 and 2 allows testing the reverse UG2UG link.

To test the AG2UG/UG2AG links, packets are transmitted from node 3 (which in this case acts as a source) to node 2, which is buried. Node 2 receives the packets and inserts the LQI and RSS for the received packets as the payload of new packets. These new packets are then transmitted back to node 3, which logs the RSS and LQI for the received packets, as well as the RSS and LQI in the payload of the same packets, where the former corresponds to the statistics of UG2AG links and the latter to the statistics of AG2UG links. The relationship between the RSS and PRR, as well as LQI and PRR, was determined using the Pearson correlation coefficient. Links in several scenarios were tested: dry UG2UG at depths of 20 and $40 \mathrm{~cm}$ for five different internode distances; wet UG2UG at a depth of $20 \mathrm{~cm}$ and internode distances of $80 \mathrm{~cm}$ and $2 \mathrm{~m}$, and dry/wet AG2UG/UG2AG for internode distances of 1-11 m. Soil samples were collected straight after each experiment was completed. It is noted that for AG2UG/UG2AG links, the internode distance corresponds to the horizontal distance between the buried node and the aboveground sink, as denoted by $d_{h 1}$ and $d_{h 2}$ in Fig. 5. Internode distances for the AG2UG and UG2AG experiments denote horizontal distances because signals in these channels do not travel in a straight path due to the refraction experienced at the medium boundaries. Additionally, the antenna height varies slightly for each position index, as the "optimal" height is determined by selecting the height which results in the maximum RSS before carrying out measurements at each position index.

Since a wave experiences angular defocusing at the soilair interface [19], the optimal height (i.e., the height at which the RSS is maximized) for the aboveground sink has to be determined. For the experimental setup described here, it was 


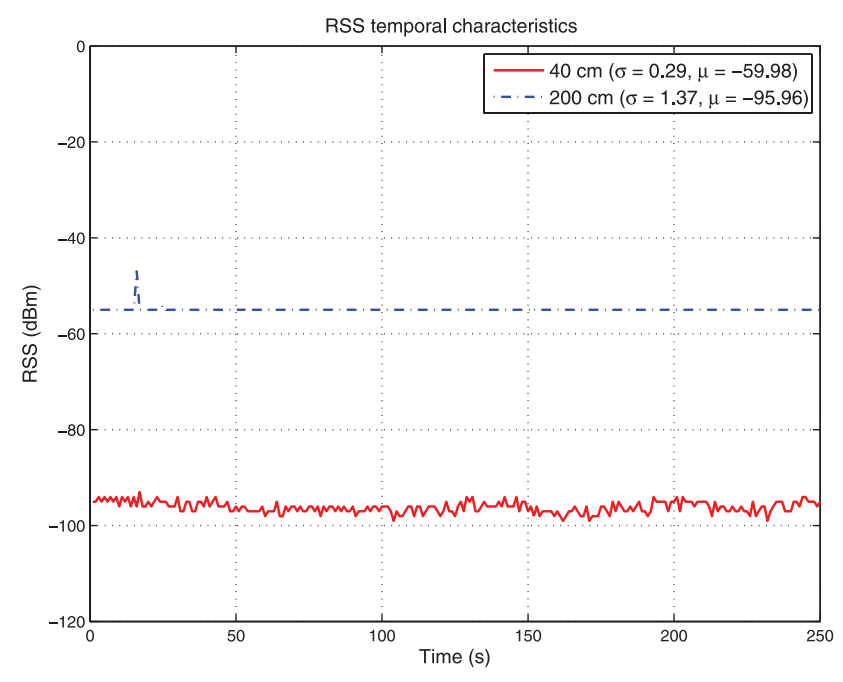

Fig. 6. UG2UG RSS temporal characteristics.

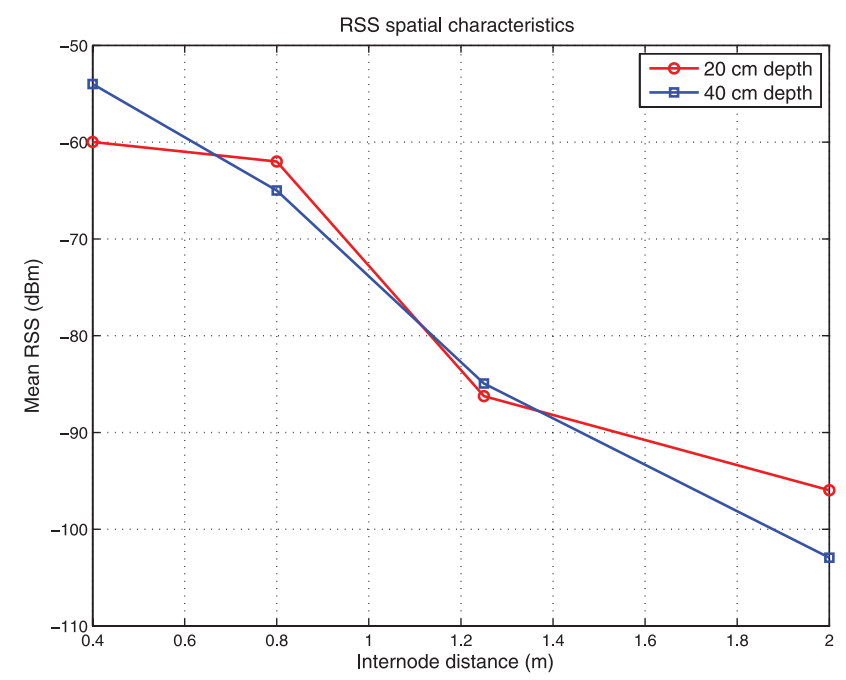

Fig. 7. UG2UG RSS spatial characteristics.

found that for both burial depths, the optimal height was approximately $1.2 \mathrm{~m}$, with a deviation of $20 \mathrm{~cm}$. Outside this range, attenuation of up to $20 \mathrm{~dB}$ was observed.

\section{Results and Discussion}

Considering the theoretical background, it is expected that communication ranges in UG2UG links will be limited due to severe attenuation (especially in wet soil conditions) and UG2AG/AG2UG links will have different degrees of attenuation at corresponding internode distances since waves propagating from soil to air will experience a higher transmission loss than waves propagating from air-to-soil [21] (due to the difference in refractive indices), and waves traveling from soil to air will experience angular defocus [19]. Furthermore, the transmission and reflection coefficients are dependent on the soil properties and will, therefore, be affected by an increase in VWC. A discussion of all results follows.

\section{A. Underground-to-Underground Channel}

The spatial characteristics of UG2UG links were determined for five horizontal internode distances at burial depths of 20 and $40 \mathrm{~cm}$.

1) RSS Temporal and Spatial Characteristics: The spatial characteristics were determined by averaging the RSS over 500 packets for each of the internode distances considered. The results for all tested UG2UG links in dry conditions are shown in Figs. 6 and 7. It is evident that UG2UG links exhibit very high temporal stability, with an RSS standard deviation of less than $1.5 \mathrm{dBm}$. It is noted that the higher RSS at $40 \mathrm{~cm}$ burial depth shown in Fig. 7 can be attributed to an optimal burial depth (as suggested in [20]), since the effect of reflections from the air-soil interface is reduced at larger burial depths, as reflected signals are attenuated to such a level that they cannot be detected at the receiver. The slightly higher standard deviation at $2 \mathrm{~m}$ is caused by the fact that the RSS is approaching the receiver sensitivity.

2) LQI Temporal and Spatial Characteristics: The LQI characteristics were measured over the same set of packets as the RSS characteristics. The results are illustrated in Figs. 8 and 9. Since the LQI in the CC430 transceiver is based on the symbol error deviation (the reader is referred to [32] for more information), the LQI will inevitably be affected by constructive and destructive interference. The LQI reported by the CC430 transceiver has a minimum value of 0 (indicating best quality) and a maximum value of 127 (indicating worst quality).

For UG2UG links, especially at shallower depths, the reflection paths can constructively or destructively interfere with the direct UG2UG path, therefore increasing the error deviation at the receiver, resulting in a lower LQI (which in the CC430 is indicated by a large LQI value). In a number of investigations reported in literature for traditional OTA WSNs, LQI has consistently exhibited a high variance [24], [26], [27]. This phenomenon is also observed here. At 2-m internode distance, the absence of reflections from the soil-air surface is negligible, resulting in a more stable LQI.

At a deeper burial depth, the effects of reflection can be reduced and the LQI will consequently change. At longer internode distances, it is possible that the attenuation experienced by the reflected signal can result in a more stable LQI at the receiver, and also result in a better mean LQI.

3) PRR and Link Asymmetry: The PRR for all tested UG2UG links is illustrated in Fig. 10. Link asymmetry is defined as the difference between the PRRs of forward and reverse links as [34]

$$
\left\|\mathrm{PRR}_{i j}-\mathrm{PRR}_{j i}\right\|
$$

where $\mathrm{PRR}_{i j}$ is the PRR for the link between nodes $i$ and $j$, and $\mathrm{PRR}_{j i}$ is the PRR for the link between nodes $j$ and $i$. The larger the difference between the PRRs, the more asymmetric the link is.

In WSNs, three communication regions exist: connected, transitional, and disconnected [35]. The connected region is the region of a link where the PRR is consistently above 0.9 ; the transitional region is the region where the PRR is between 6 


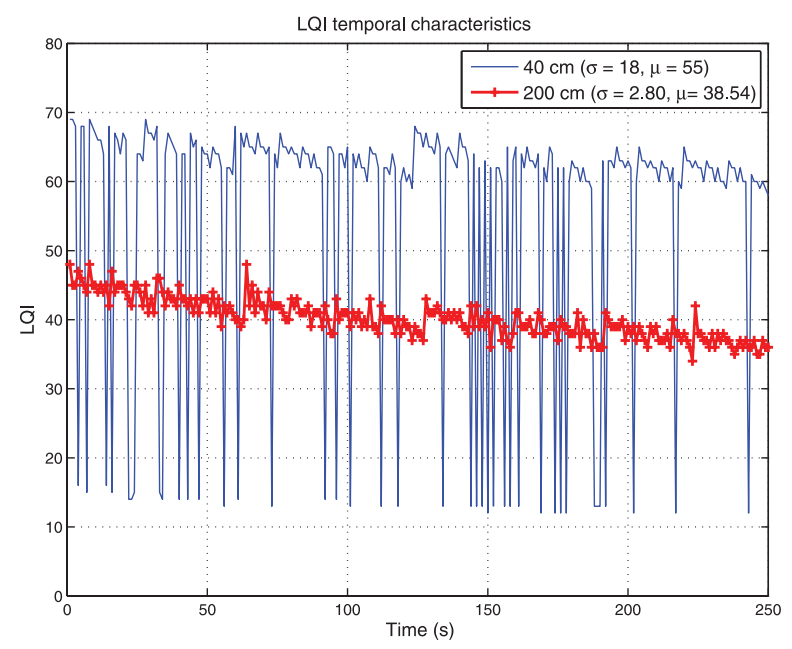

Fig. 8. UG2UG LQI temporal characteristics.

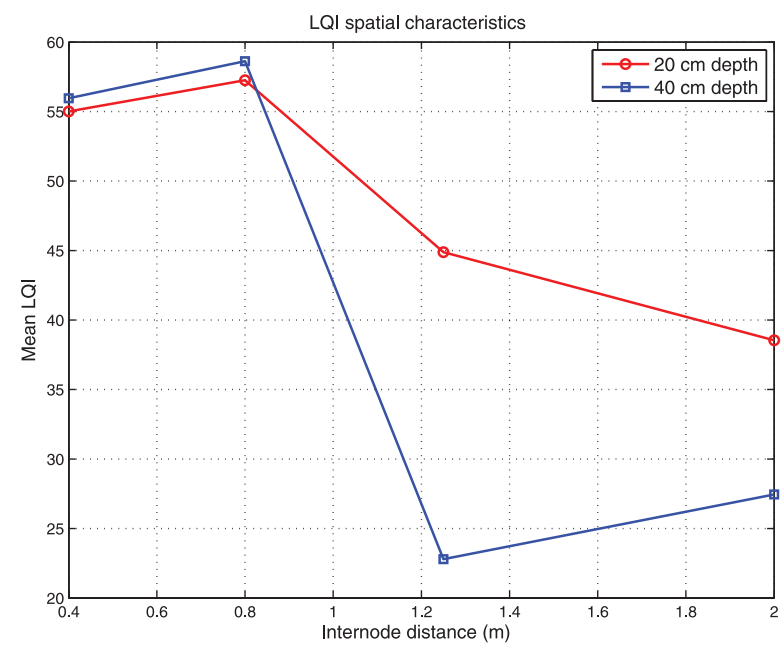

Fig. 9. UG2UG LQI spatial characteristics.

the PRR is less than 0.1. For both forward and reverse links tested, the transitional region (shown in Fig. 10) is between 1.5 and $2.4 \mathrm{~m}$, which corresponds to $36 \%$ of the total communication range (i.e., $2.5 \mathrm{~m}$ ). The connected region between 0 and $1.5 \mathrm{~m}$ is larger than the transitional region, and the disconnected region is very narrow. The asymmetry for all tested links ranges between 0 and 0.0820 . It is evident that UG2UG links are very symmetric, highly stable, and consist of mainly links in the connected region.

4) RSS, LQI, and PRR in Wet Scenario: Given the theoretical background presented in Section II, an increase in VWC results in higher path loss. Previous studies have shown a difference of up to $12 \mathrm{dBm}$ for a $7 \%$ VWC increase [23]. It is noted that in [23], the effect of the VWC on the signal's wavelength was not taken into consideration and it is, therefore, reasonable to assume that the path loss would have been less if a proper antenna had been selected.

The experimental results for the wet UG2UG scenario were only obtained at internode distances of $80 \mathrm{~cm}$ and $2 \mathrm{~m}$ at a burial depth of $20 \mathrm{~cm}$, as it was challenging to retrieve nodes buried at a depth of $40 \mathrm{~cm}$ due to muddy conditions. A comparison

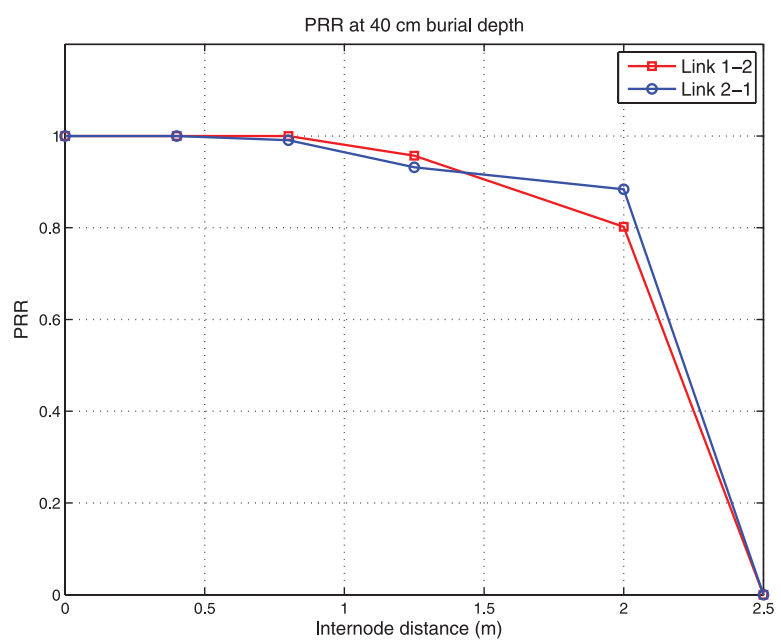

Fig. 10. Forward and reverse PRRs for UG2UG links.

TABLE III

COMPARISON OF RSS FOR WET AND DRY SCENARIOS

\begin{tabular}{|l|l|l|l|l|}
\hline $\begin{array}{l}\text { Inter-node } \\
\text { distance }(\mathrm{m})\end{array}$ & $\begin{array}{l}\text { RSS } \\
\text { Mean (wet) }\end{array}$ & $\begin{array}{l}\text { RSS } \\
\text { Std (wet) }\end{array}$ & $\begin{array}{l}\text { RSS } \\
\text { Mean (dry) }\end{array}$ & $\begin{array}{l}\text { RSS } \\
\text { Std (dry) }\end{array}$ \\
\hline 0.8 & -65.8 & 0 & -61.99 & 0.09 \\
\hline 2 & -103.8 & 0.084 & -95.96 & 1.37 \\
\hline
\end{tabular}

of the RSS characteristics in dry and wet scenarios is shown in Table III.

These results show that at $0.8 \mathrm{~m}$ there was a decrease in RSS of $3.81 \mathrm{dBm}$, and $7.84 \mathrm{dBm}$ at $2 \mathrm{~m}$. With regard to LQI, no significant changes were observed. LQI exhibited similar variance and similar spatial behavior as in the dry scenario. PRR was severely reduced (to $45 \%$ ) as the added attenuation resulted in the RSS at $2 \mathrm{~m}$ to be very close to the receiver sensitivity, thereby increasing the PER, consequently decreasing the PRR.

\section{B. Underground-to-Aboveground and Aboveground-to- Underground Channels}

In comparison to the UG2UG channel, there are some distinct differences in AG2UG/UG2AG channels. First, the AG2UG channel is expected to have worse performance than the UG2AG channel because waves are highly reflected at the air-soil interface due to the soil's higher refractive index. Furthermore, the transmission losses experienced at the medium interfaces differs for each channel due to the difference in refractive indices. For all UG2AG/AG2UG experiments, the underground node was buried at $40 \mathrm{~cm}$.

1) RSS Temporal and Spatial Characteristics: The temporal characteristics shown in Fig. 11 were obtained at an internode distance of $4 \mathrm{~m}$. The temporal variations introduced by the underground portion of the channel are very small; therefore, the temporal characteristics of the aboveground portion of the channel dominate. The transmission losses at the soil-air interface are larger than the transmission losses at the air-soil interface since the soil's refractive index is larger than the air's. Therefore, the communication range for the AG2UG channel is 


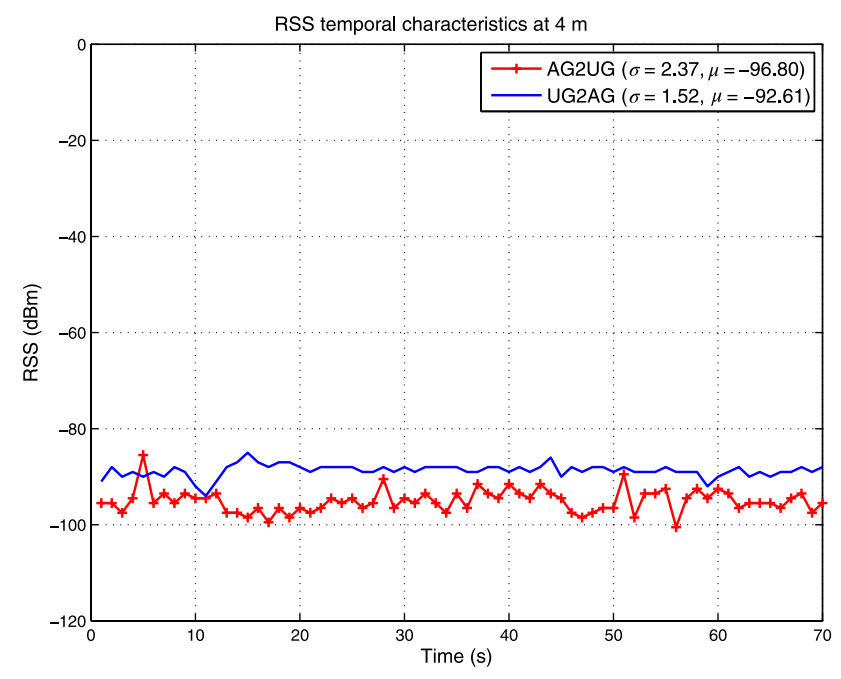

Fig. 11. UG2AG/AG2UG RSS temporal characteristics.

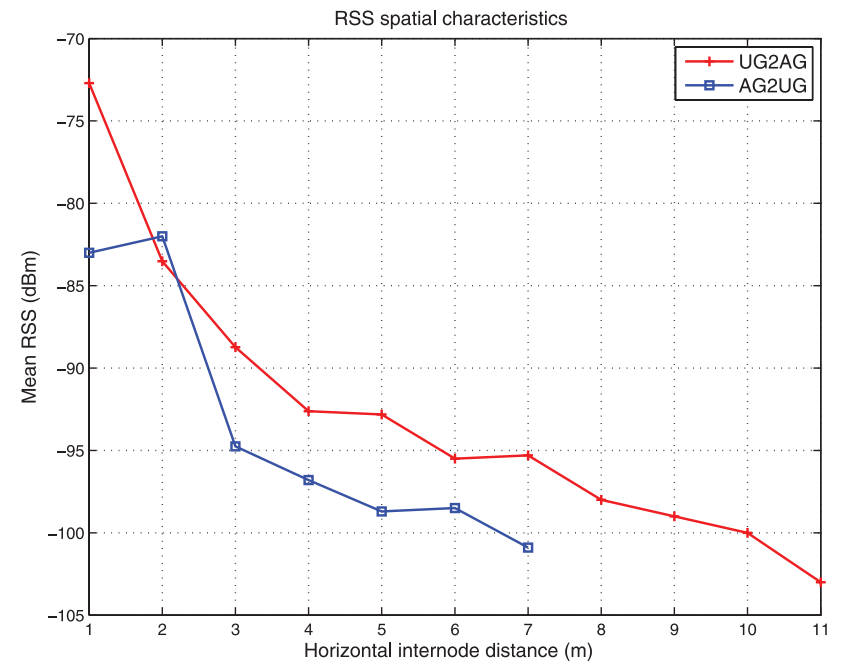

Fig. 12. UG2AG/AG2UG RSS spatial characteristics.

2) LQI Temporal and Spatial Characteristics: The LQI behavior for UG2AG and AG2UG links differs from the LQI behavior observed in UG2UG links. While in UG2UG links the mean LQI decreases with increasing distance, this behavior is not observed in UG2AG/AG2UG links (as illustrated in Fig. 13), where the LQI first increases and then decreases. This change can be partially attributed to the effects from the ground surface which is not smooth and can cause scattering, given that the aboveground node is in close proximity to the underground node. As the node is moved further away, this effect changes.

The temporal characteristics for these links exhibit less variance, possibly due to the fact that an interfering path from the UG2UG channel does not exist, as communication occurs strictly between underground and aboveground nodes. Furthermore, it is reasonable to assume that the LQI is affected by refraction, reflection, and also angular defocus. Further experimentation is required to determine the exact effect of these propagation phenomena on the LQI.

3) PRR and Link Asymmetry: The asymmetry of links in UG2AG/AG2UG channels is shown in Fig. 14.

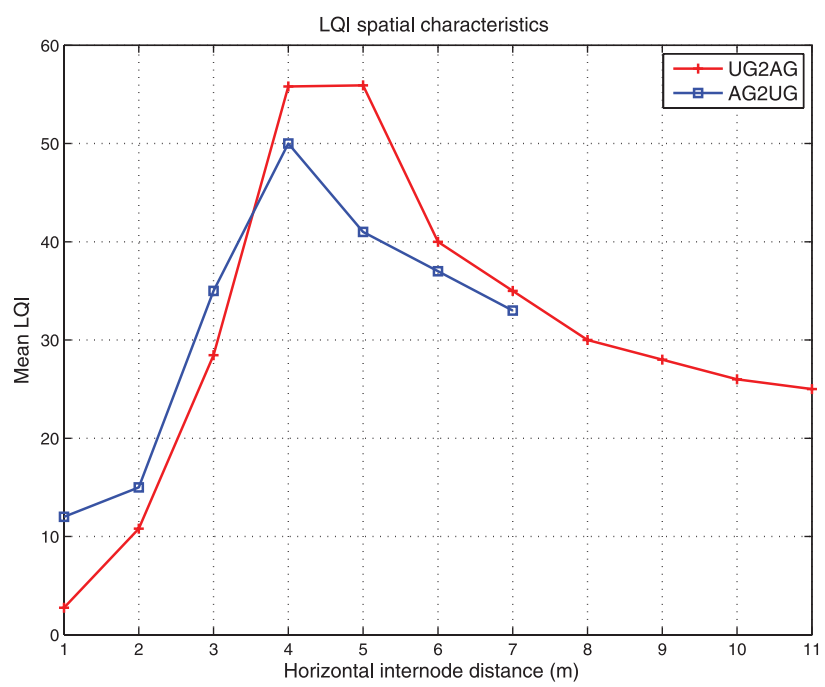

Fig. 13. UG2AG/AG2UG LQI spatial characteristics.

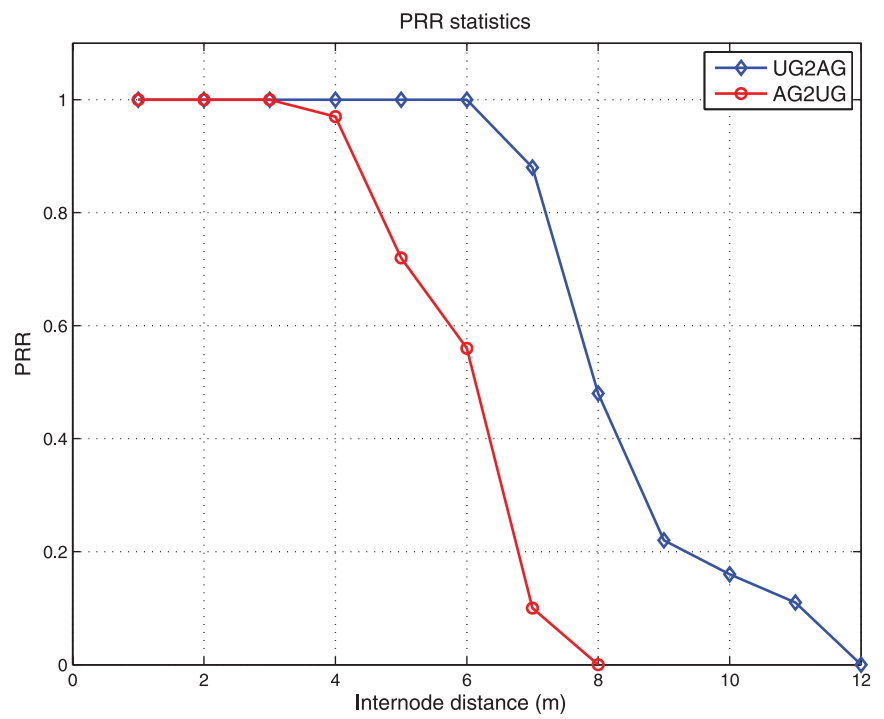

Fig. 14. PRR for UG2AG and AG2UG links.

It is shown that although the transitional regions for both channels have similar width, the connected region of the AG2UG channel is smaller due to the smaller communication range. As the communication signal is attenuated at the air-soil interface, the RSS drops to values very close to the receiver sensitivity, therefore decreasing the PRR. To determine this asymmetry, a pair-wise comparison between UG2AG/AG2UG links was performed up to $7 \mathrm{~m}$, at which point the AG2UG channel reached its maximum communication range. It was observed that the asymmetry ranged from 0 to 0.78 . In comparison to UG2UG links, where the asymmetry ranged from 0 to 0.0820 , it is evident that UG2AG/AG2UG channels are much more asymmetric.

4) RSS, LQI, and PRR in Wet Scenario: An increase in VWC results in higher path loss for links in both channels. The communication range for links in the UG2AG channel reduced by $32 \%$, while the communication range for links in the AG2UG channel reduced by $27 \%$. It is observed that the 8

RSS variance is consistent with the dry scenario; therefore, the 


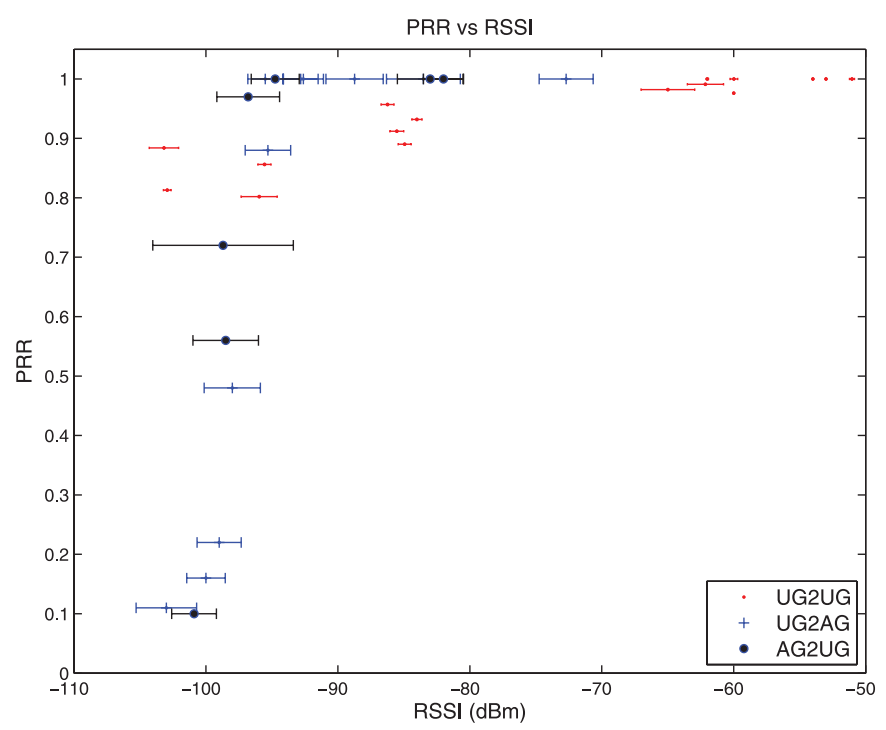

Fig. 15. PRR versus RSS.

increase in VWC has no apparent effect on the RSS temporal characteristics. However, the observed behavior for LQI differs, as the LQI mean is smaller for corresponding distances (in comparison to the dry scenario), possibly due to the change in the soil's refractive index. In the wet scenario, it is noted that there is a slight difference in transitional region widths, indicating that higher VWC decreases the asymmetry.

\section{Relationship Between RSS, LQI, and PRR}

It is of interest to determine the relationship between PRR, RSS, and LQI. In particular, since both RSS and LQI are hardware-based estimators (and therefore simpler to use), using either as an indicator of PRR is a more efficient approach than estimating PRR directly.

The relationship between the three parameters was determined by computing the Pearson correlation coefficient between the mean RSS and PRR, and mean LQI and PRR for all channels. Fig. 15 illustrates the RSS-PRR correlation. The three correlation coefficients are $0.9092,0.6879$, and 0.6551 for the UG2UG, UG2AG, and AG2UG channels, respectively. Fig. 16 illustrates the LQI-PRR correlation.

For LQI-PRR, the Pearson coefficients are 0.2000, 0.1247, and 0.1673 for the UG2UG, UG2AG, and AG2UG channels, respectively. This shows that in contrast to RSS, which is highly correlated with PRR, the LQI-PRR correlation is much lower for all three channels. Unlike other studies of WSNs where mean LQI has been shown to be a good indicator of PRR (the reader is referred to [24] for more information), this is not the case for WUSNs. This partly stems from the fact that LQI is a much more sensitive metric in comparison to RSS, and is therefore prone to effects from propagation (reflection and refraction for instance) as well as interference.

In the CC430 transceiver, the LQI "accumulates the magnitude of the error between the ideal constellations and received signal over 64 symbols immediately following the sync word" [32]. Since EM waves undergo a change in wavelength when travelling from soil to air (and air to soil) and undergo some transmission loss (among other effects), certain properties of

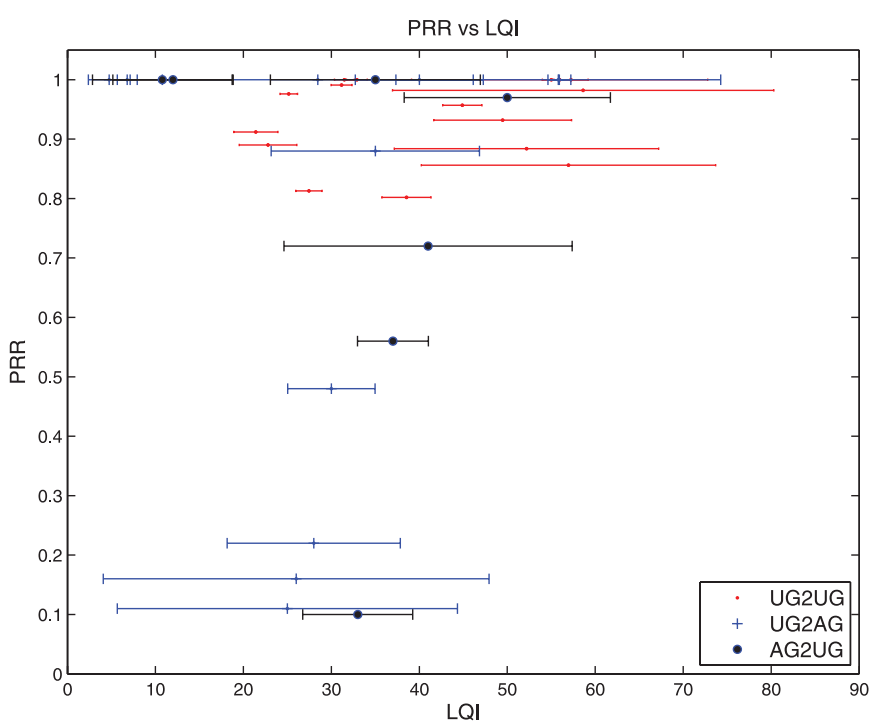

Fig. 16. PRR versus LQI.

the wave such as phase or amplitude can be affected. Therefore, it is reasonable to assume that the LQI will be adversely affected as these effects will "corrupt" the original signal, making it more difficult for the receiver to decode the signal, resulting in a poorer LQI. Furthermore, scattering can be caused by rocks and roots on the ground surface and the fact that the surface is not smooth can result in multiple paths (which can potentially interfere) reaching the receiver. Overall, in contrast to the general trait in aboveground WSNs where LQI is typically a better indicator of PRR, it is seen that in WUSNs, the RSS has higher correlation with PRR, and is therefore a better indicator of PRR than LQI.

\section{Summary of Results}

Several experiments were performed to evaluate the temporal and spatial characteristics of all three selected link quality metrics. Links in the UG2UG channel exhibit high-quality traces (i.e., extreme RSS high temporal stability and low link asymmetry), but are subject to limited communication ranges. An increase in VWC does not affect the temporal characteristics for RSS or LQI in UG2UG links. The LQI is highly variant and not correlated with PRR, and its mean decreases with increasing internode distance for both dry and wet conditions in UG2UG links.

The temporal characteristics for the UG2AG/AG2UG links are comparable to OTA links. It is observed that for these links, the LQI differs between dry and wet scenarios, due to the effects of the air-soil and soil-air interfaces which are affected by the soil's VWC. Further modeling and experimentation is required to determine the actual effect the medium interfaces have on the LQI. For all three channels, it was observed that the RSS is a better indicator of PRR, with the highest correlation between RSS and PRR observed in the UG2UG channel.

\section{E. Impact of This Work}

The results of this study have some evident consequences on protocol development. The high stability of the UG2UG 
channel can be exploited for fast link quality estimation, where RSS-based link quality estimation with a single packet is sufficient to select forwarding links. Additionally, this high temporal stability enables very stable topologies, therefore reducing the network overhead required for topology establishment, and consequently reducing energy consumption.

1) Development of Novel Communication Protocols Robust to Asymmetry and Environmental Conditions: For UG2AG/AG2UG channels, the inherent asymmetry can be exploited by metrics which favor uni-directional links, such as Expected number of Transmissions over Forward links (ETF) [36], since PRR-based metrics such as expected transmission count (ETX) discriminate against highly asymmetric links. Furthermore, environmental awareness has to be integrated into communication protocols for WUSNs. For instance, transmit power can be minimized in dry scenarios, but the WUSN has to be aware of the soil's moisture content to trigger such an action. There is existing work [37] which can be extended for these purposes. Given the different characteristics of the three channels, it is evident that highly adaptive protocols robust to environmental conditions are required for efficient communication in WUSNs.

Additionally, relationships between RSS, LQI, and PRR presented in this work demonstrated an interesting phenomenon: unlike conventional WSNs, the RSS in WUSNs has a higher correlation with PRR than LQI [24]. This means that communication protocols which exploit the correlation between LQI and PRR are not useful for WUSNs. The link quality characteristics presented in this paper give researchers an insightful basis to work from when developing novel communication protocols specifically tailored for WUSNs.

2) Guidelines for Further Experimentation: It is evident that further investigation into properties of LQI should be conducted, as the LQI behavior observed in this study was not consistent. Ideally, experiments which make use of spectrum analyzers can offer greater insight into the properties of LQI, and analysis of the channel impulse response (CIR) of received signals in WUSNs can clarify some aspects related to LQI behavior. This will be addressed in future work.

\section{Related Work}

Several studies have investigated wireless propagation in soil. In [9], [21], and [33], experimental channel characterizations were performed in test beds for UG2UG, AG2UG, and UG2AG channels in WUSNs. Theoretical propagation models for all three channels were proposed in [18] and [20], and near-surface effects from disturbances on channel characteristics were presented in [38], as well as network connectivity in [39]. An overview of experimental studies on WUSNs is shown in Table IV.

Some of these propagation models have been verified in experiments such as in [33], where characteristics of the UG2UG channel were analyzed using Mica2 nodes at $433 \mathrm{MHz}$, and guidelines for test-bed implementation were given. The UG2AG channel was investigated in [19], where soil scout (a custom built $868 \mathrm{MHz}$ sensor node) was proposed for soil moisture monitoring. Both UG2AG and AG2UG
TABLE IV

OVERVIEW OF STUDIES ON WUSNS

\begin{tabular}{c||c|c} 
Reference & Radio frequency $(\mathrm{MHz})$ & Channel \\
\hline$[17]$ & 433 & UG2UG \\
\hline$[19]$ & 868 & UG2AG \\
\hline$[21]$ & 433 & UG2AG/AG2UG \\
\hline$[33]$ & 433 & UG2UG
\end{tabular}

channels were experimentally investigated in [21]. It is evident that most of these studies have been typically carried out separately (i.e., no single study investigated both UG2UG and UG2AG/AG2UG channels simultaneously). Therefore, it is reasonable to assume that some aspects of the experimental setup (e.g., antenna configuration) might only be applicable to a specific type of channel. In some studies (such as [33]), the antenna selection was not considered, and in some cases, the propagation path for the UG2UG channel was not strictly soil to soil, as paper pipes were used in [33] as containers for buried sensor nodes, and in some cases, nodes were placed in a solid container before burial [17]. Therefore, it is evident that none of these studies use the same antenna configuration for all three channels. This poses a challenge because real WUSN deployments will typically have a single antenna per node; therefore, it is crucial to characterize all channels using the same type of antenna in all buried nodes.

The work in this paper is an extension of the work presented in [21] and [33], where the UG2UG and UG2AG/AG2UG channels were analyzed separately. This extends both studies by conducting experiments with a single experimental setup, and also by analyzing the relationships of RSS and PRR and PRR and LQI, which has never been done in previous theoretical nor experimental studies. The knowledge presented in this study is fundamental to understanding link quality characteristics of WUSNs. Based on these results, novel communication protocols which exploit the characteristics of RSS, LQI, and PRR (and the relationship between these parameters) can now be developed. Characteristics presented in previous studies are valid, and our work builds on them. For instance, our experiments show that although the UG2AG/AG2UG channel is asymmetric (which was also observed in [21]), the UG2UG channel is highly symmetric (which had not been observed before). This means that communication protocols which accommodate a mixture of highly symmetric and asymmetric links efficiently have to be developed, and the work in this paper is fundamental to the development of such protocols.

\section{Conclusion}

Link quality was characterized for the three channels in WUSNs. The results show that links in the UG2UG channel are temporally stable, with high PRRs, but with limited communication ranges. On the other hand, links in UG2AG/AG2UG channels exhibit temporal characteristics comparable to OTA channels and are sensitive to changes in soil conditions. Although UG2UG links exhibit high quality traces, the limited range limits the use of this channel. Therefore, research efforts should be more focused on the UG2AG/AG2UG channels to 10 
signals at the soil-air and air-soil interfaces (in both dry and wet conditions) as well as modeling LQI behavior. It was also shown that RSS has a higher correlation with PRR than LQI in all three channels. The results of this work can be used as a basis for development of novel communication protocols for WUSNs, which are robust to link asymmetry and environmental conditions.

\section{References}

[1] K. Derr and M. Manic, "Wireless sensor networks—Node localization for various industry problems," IEEE Trans. Ind. Informat., vol. 11, no. 3, pp. 752-762, Jun. 2015.

[2] A. Kumar, H. Kim, and G. P. Hancke, "Environmental monitoring systems: A review," IEEE Sensors J., vol. 13, no. 4, pp. 1329-1339, Apr. 2013.

[3] P. Corke, T. Wark, R. Jurdak, W. Hu, P. Valencia, and D. Moore, "Environmental wireless sensor networks," Proc. IEEE, vol. 98, no. 11, pp. 1903-1917, Nov. 2010.

[4] G. P. Hancke, B. Silva, and G. P. Hancke Jr., "The role of advanced sensing in smart cities," MDPI Sensors, vol. 13, no. 1, pp. 393-425, Dec. 2013.

[5] A. Mainwaring, D. Culler, J. Polastre, R. Szewczyk, and J. Anderson, "Wireless sensor networks for habitat monitoring," in Proc. 1st ACM Int. Workshop Wireless Sensor Netw. Appl. (WSNA), 2002, pp. 88-97.

[6] G. P. Hancke and G. P. Hancke Jr., "Industrial wireless sensor networks: A selection of challenging applications," in Proc. 6th Eur. Conf. Antennas Propag. (EuCAP), pp. 64-68, Mar. 26-30, 2012.

[7] S. Villingiri, A. Ray, and M. Kande, "Wireless infrastructure for oil and gas inventory management," in Proc. 39 Аnпи. Conf. IEEE Ind. Electron. Soc. (IECON), Nov. 2013, pp. 5461-5466.

[8] V. C. Gungor and G. P. Hancke, "Industrial wireless sensor networks: Challenges, design principles, and technical approaches," IEEE Trans. Ind. Electron., vol. 56, no. 10, pp. 4258-4265, Oct. 2009.

[9] L. Tang, K. Wang, Y. Huang, and F. Gu, "Channel characterization and link quality assessment of IEEE 802.15.4-compliant radio for factory environments," IEEE Trans. Ind. Informat., vol. 3, no. 2, pp. 99-110, May 2007.

[10] L. Tang, K. Wang, and Y. Huang, "Study of speed-dependent packet error rate for wireless sensor on rotating mechanical structures," IEEE Trans. Ind. Informat., vol. 9, no. 1, pp. 72-80, Feb. 2013.

[11] B. Silva and G. P. Hancke, "On link quality aware routing for industrial wireless sensor networks," in Proc. IEEE Int. Conf. Ind. Technol. (ICIT), pp. 1347-1352, Feb. 2013.

[12] P. Gaj, J. Jusperneite, and M. Felser, "Computer communication within industrial distributed environment," IEEE Trans. Ind. Informat., vol. 9, no. 1, pp. 182-189, Feb. 2013.

[13] A. Grilo, J. Chen, M. Diaz, D. Garrido, and A. Casaca, "An integrated WSAN and SCADA system for monitoring a critical infrastructure," IEEE Trans. Ind. Informat., vol. 10, no. 3, pp. 1755-1764, Aug. 2014.

[14] C. Potter, G. P. Hancke, and B. Silva, "Machine-to-machine: Possible applications in industrial networks," in Proc. IEEE Int. Conf. Ind. Technol. (ICIT), Feb. 2013, pp. 1321-1326.

[15] L. Da Xu, W. He, and S. Li, "Internet of Things in industries: A survey," IEEE Trans. Ind. Informat., vol. 10, no. 4, pp. 2233-2243, Nov. 2014.

[16] E. Ancillotti, R. Bruno, and M. Conti, "Reliable data delivery with the IETF routing protocol for low-power and lossy networks" IEEE Trans. Ind. Informat., vol. 10, no. 3, pp. 1864-1877, Aug. 2014.

[17] S. Yoon, L. Cheng, E. Ghazanfari, S. Pamakcu, and M. Suleiman, "A radio propagation model for wireless underground sensor networks," in Proc. IEEE Global Telecommun. Conf. (GLOBECOM), Dec. 5-9, 2011, pp. $1-5$.

[18] H. Xiaoya, G. Chao, W. Bingwen, and X. Wei, "Channel modeling for wireless underground sensor networks," in Proc. IEEE 35th Annu. Comput. Softw. Appl. Conf., Jul. 18-22, 2011, pp. 249-254.

[19] J. Tiusanen, "Attenuation of a soil Scout radio signal," Elsevier Biosyst. Eng., vol. 90, no. 2, pp. 127-133, 2005.

[20] L. Li and I. F. Akyildiz, "Characteristics of underground channel for wireless underground sensor networks," in Proc. 6th Annu. Mediterr. Ad Hoc Netw. Workshop, 2007, pp. 92-99.

[21] A. Silva and M. C. Vuran, "Communication with aboveground devices in wireless underground sensor networks: An empirical study," in Proc. IEEE Int. Conf. Commun. (ICC), May 23-27, 2010, pp. 1-6.

[22] H. Karl and A. Willig, Protocols and Architecture for Wireless Sensor Networks. Hoboken, NJ, USA: Wiley, 2006.
[23] D. Lymberoupoulos, Q. Lindsey, and A. Savvides, "An empirical characterization of radio signal strength variability in 3-d IEEE 802.15.4 networks using monopole antennas," in Proc. 3rd Eur. Conf. Wireless Sensor Netw. (EWSN), 2006, pp. 326-341.

[24] N. Baccour, A. Koubaa, M. Zuniga, H. Youssef, C. Boano, and M. Alves, "Radio link quality estimation in wireless sensor networks: A survey," ACM Trans. Sensor Netw., vol. 8. no. 4, pp. 1-33, Sep. 2012.

[25] A. Vlavianos, L. Law, L. Broustis, S. Krishnamurthy, S. Faloutsos, and Michalis, "Assessing link quality in wireless networks: Which is the right metric?," in Proc. IEEE 19th Int. Symp. Pers. Indoor Mobile Radio Commun., Sep. 15-18, 2008, pp. 1-6.

[26] C. Boano et al., "Exploiting the LQI variance for rapid channel quality assessment," in Proc. Int. Conf. Inf. Process. Sensor Netw. (IPSN), Apr. 13-16, 2009, pp. 369-370.

[27] C. Boano, M. Zuniga, T. Voigt, A. Willig, and K. Romer, "The triangle metric: Fast link quality estimation for mobile wireless networks," in Proc. 19th Int. Conf. Comp. Commun. Netw. (ICCCN), pp. 1-7, Aug. 2-5, 2010.

[28] I. Jawhar, N. Mohamed, M. Mohamed, and J. Aziz, "A routing protocol and addressing scheme for oil, gas, and water pipeline monitoring using wireless sensor networks," in Proc. Int. Conf. Wireless Opt. Commun. Netw. (WOCN), 2008, pp. 1-5.

[29] I. F. Akyildiz and M. C. Vuran, Wireless Sensor Networks. Hoboken, NJ, USA: Wiley, 2010.

[30] H. M. Ammari, The Art of Wireless Sensor Networks Volume 2: Advanced Topics and Applications. New York, NY, USA: Springer, 2014.

[31] N. Peplinski, F. Ulaby, and M. Dobson, "Dielectric properties of soils in the 0.3-1.3 GHz range," IEEE Trans. Geosci. Remote Sens., vol. 33, no. 3 , pp. 803-807, May 1995.

[32] CC1101 Sub 1-GHz Transceiver datasheet, Texas Instruments [Online]. Available: http://www.ti.com/lit/ds/symlink/cc1101.pdf

[33] A. Silva and M. C. Vuran, "Development of a testbed for wireless underground sensor networks," EURASIP J. Wireless Commun. Netw., vol. 2010, pp. 1-14, 2010.

[34] J. Zhao and R. Govindan, "Understanding packet delivery performance in dense wireless sensor networks," in Proc. 1 st Int. Conf. Embedded Netw. Sensor Syst. (SenSys), Nov. 5-7, 2003, pp. 1-13.

[35] M. Zuniga and B. Krishnamachari, "Analyzing the transitional region in low power wireless links," in Proc. 1st Annu. IEEE Commun. Soc. Conf. Sensor Ad Hoc Commun. (SECON), Oct. 4-7, 2004, pp. 517-526.

[36] L. Sang, A. Arora, and H. Zhang, "On link asymmetry and one-way estimation in wireless sensor networks," ACM Trans. Sensor Netw., vol. 6, no. 2, pp. 1-25, Feb. 2010.

[37] S. Fang et al., "An integrated system for regional environmental monitoring and management based on Internet of Things," IEEE Trans. Ind. Informat., vol. 10, no. 2, pp. 1596-1605, May 2014.

[38] L. Li, W. Wu, J. Chen, and Y. Liu, "Interference of human's ankle to the channel characteristics of near surface WUSN," J. China Univ. Posts Telecommun., vol. 21, no. 1, pp. 36-42, Feb. 2014.

[39] Z. Sun, I. F. Akyildiz, and G. P. Hancke, "Dynamic connectivity in wireless underground sensor networks," IEEE Trans. Wireless Commun., vol. 10, no. 12, pp. 4332-4344, Dec. 2011.

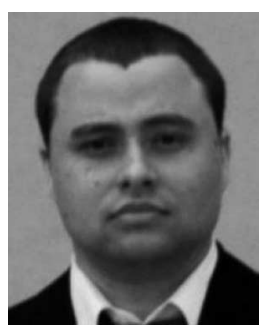

Bruno Silva (S'14) received the B.Eng., B.Eng. (Hons.), and M.Eng. degrees in computer engineering from the University of Pretoria, Pretoria, South Africa, in 2011, 2012, and 2015, respectively.

From September 2013 to February 2014, he was a Visiting Researcher at the ABB Corporate Research Centre, Västerås, Sweden, where he was involved in research on ultra wideband based localization. His research interests include wireless sensor networks, digital signal processing, and mobile computing.

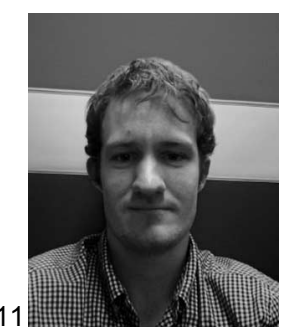

Roy M. Fisher (S'14) received the B.Eng. and Hons degrees in computer engineering from the University of Pretoria, Pretoria, South Africa, in 2012 and 2013, respectively, where he is currently pursuing the M.Eng. degree in computer engineering with his primary research area being the Internet of Things.

His research interests include embedded systems, wireless sensor networks, and data security. 


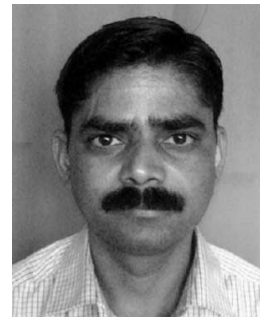

Anuj Kumar received the Ph.D. degree in embedded systems from the Indian Institute of Technology Delhi, New Delhi, India, in 2011; the M.Tech degree in instrumentation from the National Institute of Technology Kurukshetra, Kurukshetra, India, in 2004; and the M.Phil. degree in instrumentation from the Indian Institute of Technology Roorkee, Roorkee, India, in 2000

He was with the APL Intelligent Embedded, New Delhi, where he was involved in the development of microcontroller-based applications from 2000 to 2002. In 2004, he joined DellSoft Technologies, New Delhi, as an Instrumentation Engineer EDA. From January 2012 to October 2102, he worked as a Postdoctoral Research Fellow with the Department of Electrical and Computers Engineering, University of Seoul, Seoul, South Korea. Since November 2012, he has been a Vice-Chancellor Post Doctoral Fellow with the Department of Electrical, Electronic, and Computer Engineering, University of Pretoria, Pretoria, South Africa. His research interests include intelligent systems, smart building, and instrumentation electronics.

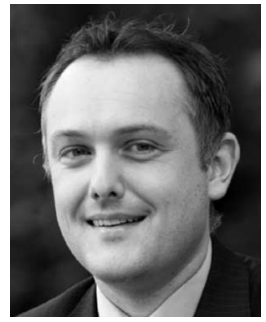

Gerhard P. Hancke (S'99-M'07-SM'11) received the B.Eng. and M.Eng. degrees in computer engineering from the University of Pretoria, Pretoria, South Africa, in 2002 and 2003, respectively, and the $\mathrm{Ph} . \mathrm{D}$. degree in computer science with the security group from the University of Cambridge's Computer Laboratory, Cambridge, U.K., in 2008.

$\mathrm{He}$ is an Assistant Professor with the City University of Hong Kong, Hong Kong. His research interests include system security, embedded platforms, and distributed sensing applications. 